\title{
Inner Approximations of Consistent Parameter Sets by Constraint Inversion and Mixed-Integer Programming
}

\author{
Stefan Streif \\ Nadine Strobel \\ Institute for Automation Engineering, \\ Department for Systems Theory and Automatic Control, \\ Otto-von-Guericke University Magdeburg, Germany
}

Rolf Findeisen

\begin{abstract}
Mathematical modeling has become an indispensable tool in the analysis, prediction and control of chemical and biological systems. However, the estimation of consistent model parametrizations and model invalidation are challenging tasks, but crucial for reliable modelbased analysis and prediction. Set-based estimation methods are useful to determine guaranteed outer approximations of consistent parameter sets, i. e. consistent parametrizations are never excluded. However, these conservative outer approximating sets often include inconsistent parametrizations which lead to inconsistent models and hence possibly wrong model-based predictions. This paper proposes a set-based framework to determine inner approximations, i. e. the model is guaranteed consistent with measurement data for all parametrizations from this set. Our approach is based on the reformulation and inversion of measurement data constraints and by imposing nonlinear constraints on binary variables. The relaxation of the mixed-integer nonlinear feasibility problem into a mixed-integer linear feasibility problem allows the inner approximations to be determined efficiently. The applicability of this approach is demonstrated considering a nonlinear biochemical reaction network.
\end{abstract}

Keywords: Estimation; Model verification; Dynamic modeling; Convex optimization.

\section{INTRODUCTION}

In many applications, from chemical engineering to systems biology, mathematical models are crucial for the analysis, design and control of the considered processes. When building a model, parameters are often unknown and cannot be measured directly, but have to be estimated from experimental data. Often, however, it is not sufficient to estimate just one model parameterization that minimizes an objective function like model output and data deviation. Instead one might be interested in parameter bounds or sets for which the experimental data can be reproduced. For this purpose, set-based model invalidation and estimation methods are useful [Rumschinski et al., 2010, Borchers et al., 2009].

Within a set-based estimation framework, measurement data and parameter uncertainties can be taken directly into account and outer approximations of consistent parameter sets can be obtained. These outer approximations are guaranteed to include all consistent parameter values (cf. Fig. 1a). However, due to the conservatism of set-based methods and the outer approximation approach, inconsistent parameter combinations can still be included in the determined sets. It is therefore desired to determine inner approximations, i. e. sets for which it is guaranteed that all parameter values from the set are guaranteed consistent. Even in the presence of uncertainties, this enables one

1 Corresponding author: Stefan Streif. E-mail addresses: \{stefan.streif, nadine.strobel, rolf.findeisen\}@ovgu.de. to make reliable model-based predictions, which play an important role e.g. in control and therapy design.

This work proposes an approach for the estimation of inner approximations by means of an inverted reformulation of constraints and their logical combination into a mixed-integer nonlinear feasibility problem. Inner approximations and set-inversions have been considered before in the systems and control community. Jaulin and Walter [1993] proposed an algorithm using set inversion and interval analysis. Methods from robust control are often employed to determine inner approximations [Zhou et al., 1995]. Henrion and co-workers developed methods for outer and inner approximations of region-of-attractions and consistent parameter sets of continuous-time systems with occupation measures [Korda et al., 2012, Streif et al., 2013].

In this work, inner approximations of the a-priori unknown consistent parameter sets for nonlinear discrete-time systems are derived. The problem is stated in Section 2. For this, constraints on data and variables are reformulated using logical operators and constraint inversion. This allows certificates on guaranteed consistent parameter sets to be determined (Section 3) by checking infeasibility of a mixedinteger nonlinear feasibility problem. Linear relaxations are employed to solve the problems efficiently and two algorithms are presented to derive inner approximations (Section 4). The approach is applied to an example of a biochemical reaction network in Section 5 . 


\section{PROBLEM SETUP}

This section introduces the considered system class and uncertainties, and states the problem of consistent parameter estimation.

\subsection{Models and Uncertainties}

Consider polynomial discrete-time systems given by

$$
\begin{aligned}
F(x(k+1), x(k), p) & =0 \\
H(y(k), x(k), p) & =0,
\end{aligned}
$$

where $F: \mathbb{R}^{n_{x}} \times \mathbb{R}^{n_{x}} \times \mathbb{R}^{n_{p}} \rightarrow \mathbb{R}^{n_{x}}$ are polynomial functions, $x(k) \in \mathbb{R}^{n_{x}}$ is the time-dependent state vector, and $p \in \mathbb{R}^{n_{p}}$ the time-invariant parameter vector. Time is indexed by $k \in \mathbb{N}$. The model output equations are given by $H: \mathbb{R}^{n_{y}} \times \mathbb{R}^{n_{x}} \times \mathbb{R}^{n_{p}} \rightarrow \mathbb{R}^{n_{y}}$, which are assumed polynomial functions, and $y(k) \in \mathbb{R}^{n_{y}}$ denote the timedependent model outputs. Such a discrete-time model (1) can be obtained from a continuous-time model by numerical integration schemes.

For parameter estimation and validation, we assume that $n_{t}$ different measurements are taken at time-instances indexed by $k \in \mathcal{T}:=\left\{0,1, \ldots, n_{t}-1\right\}$. For easier notation, let $m_{y}=\left[y_{1}(0), \ldots, y_{1}\left(n_{t}-1\right), \ldots, y_{n_{y}}(0), \ldots, y_{n_{y}}\left(n_{t}-\right.\right.$ $1)]^{\mathrm{T}} \in \mathbb{R}^{n_{y} n_{t}}$ be the vector of all output measurements taken at the different time-instances. Typically, the measurements are uncertain. This work assumes that the uncertainties can be represented by polynomial inequality constraints in the following form

$$
\mathcal{M}:=\left\{m_{y}: g_{y, i}\left(m_{y}\right) \geq 0, i=1,2, \ldots, c_{m}\right\} \subseteq \mathcal{Y},
$$

where $c_{m}$ is the number of inequalities, and $\mathcal{Y}$ is assumed a bounded subset in $\mathbb{R}^{n_{y} n_{t}}$. To shorten the notation, we write $m_{y} \in \mathcal{M}$ to express that the measurements satisfy all inequality constraints in (2).

In addition, we assume that initial knowledge on the parameter $p$ and state values $x(k)$ is available. Let this knowledge be given by $c_{p}$ (resp. $c_{x}$ ) polynomial constraints:

$$
\begin{aligned}
& \mathcal{P}:=\left\{p: g_{p, i}(p) \geq 0, i=1,2, \ldots, c_{p}\right\} \subset \mathbb{R}^{n_{p}}, \\
& \mathcal{X}:=\left\{x: g_{x, i}(x) \geq 0, i=1,2, \ldots, c_{x}\right\} \subset \mathbb{R}^{n_{x}} .
\end{aligned}
$$

Here, $g_{p}(\cdot)$ and $g_{x}(\cdot)$ are polynomial functions and could, e.g. correspond to simple upper and lower bounds. Typically, $\mathcal{X}$ and $\mathcal{P}$ can be derived from initial knowledge or conservative assumptions about the investigated processes, or from the physical meaning of the variables (e.g. concentrations have to be non-negative).

\subsection{Estimating Consistent Parameter Sets}

This contribution considers a set-based approach to parameter estimation using the following notion:

Definition 1. (Model consistency). Model (1) is said to be consistent with the measurements $\mathcal{M}$, if there exist $p \in \mathcal{P}$ and $x(k) \in \mathcal{X} \forall k \in \mathcal{T}$, such that $m_{y} \in \mathcal{M}$.

The general aim of set-based parameter estimation is not to estimate the best parameter values $p \in \mathcal{P}$ that minimize e.g. the deviation between measurements and model output. Instead, set-based estimation aims to determine the entire set or outer bounds of parameters $\mathcal{P}_{c} \subseteq \mathcal{P}$ for which the model is consistent according to Definition 1 (see also Fig. 1a).
To check model consistency, consider the following feasibility problem (FP) which aims to find any feasible solution since optimality does not matter:

$$
\mathrm{FP}:\left\{\begin{aligned}
\text { find } & \xi_{\mathrm{FP}} \\
\text { s.t. } & \text { equations }(1), \\
& x(k) \in \mathcal{X}, \forall k \in \mathcal{T}, \\
& m_{y} \in \mathcal{M}, \\
& p \in \mathcal{P},
\end{aligned}\right.
$$

where $\xi_{\mathrm{FP}} \in \mathbb{R}^{\left(n_{x}+n_{y}\right) n_{t}+n_{p}}$ is a vector that contains all the variables in (1), i. e. state and output variables at the time-instances $k \in \mathcal{T}$, and the parameters $p$.

The set of consistent parameters $\mathcal{P}_{c}$ is difficult to derive owing to the nonlinearities and nonconvexities. As previously shown [Borchers et al., 2009], it is possible to relax the FP into a convex semi-definite feasibility problem (SDP). Relaxation here refers to the fact that the consistent parameter set $\mathcal{P}_{c}$ of $\mathrm{FP}$ is fully contained in the solution set of the SDP, i. e. $\mathcal{P}_{c} \subseteq \mathcal{P}_{c}^{\mathrm{SDP}}$.

To deal with larger problems the SDP can be further relaxed into a linear program (LP), and it holds $\mathcal{P}_{c} \subseteq$ $\mathcal{P}_{c}^{\mathrm{SDP}} \subseteq \mathcal{P}_{c}^{\mathrm{LP}}$. Consistency can then be checked efficiently using the Lagrangian-dual of the linear relaxation. The weak-duality theorem and the relaxation process guarantee that if the objective of the dual program is unbounded, then FP does not admit a solution [Borchers et al., 2009].

\subsection{Outer Approximations}

While the relaxations introduce some conservatism, they allow guaranteed outer approximations of consistent parameter sets or model states, or to prove inconsistency of entire parameter regions or models. Proofs of inconsistency are particularly helpful in applications like systems biology where often competing model hypotheses exist that can then be ruled out.

Two conceptually different algorithms (cf. Fig. 1b) have been suggested to obtain outer approximations $\mathcal{P}_{o}$. The first algorithm outer-approximates $\mathcal{P}_{c}$ by sequentially and iteratively tightening the lower $\left(\underline{p}_{j}\right)$ and upper bounds $\left(\bar{p}_{j}\right)$ on single parameters $p_{j}$, which then gives an hyperrectangular outer approximation of the consistent parameter set. The second approach is to divide the parameter space $\mathcal{P}$ into partitions and to check each partition for consistency. Using a recursive algorithm like bisectioning, $\mathcal{P}_{c}$ can be approximated systematically and up to a chosen precision [Borchers et al., 2009, Streif et al., 2009, Rumschinski et al., 2010, Streif et al., 2012].

\subsection{Inner Approximations}

On the one hand, outer approximations allow the invalidation of entire models and parameter regions. But on the other hand, outer approximations are also conservative and usually contain inconsistent parameters (cf. Fig. 1). Using parameters from the derived outer approximations may therefore lead to wrong or arbitrary model-based predictions. This might be less problematic during the model building process, but it might be problematic whenever model-based predictions are important, such as in control or therapy design. 
This contribution aims to determine sets that are guaranteed to only include consistent parameter sets. We refer to these sets as inner approximations $\mathcal{P}_{i} \subseteq \mathcal{P}_{c}$.

Problem 1. (Guaranteed consistent parameter sets). Derive an inner approximation $\mathcal{P}_{i}$ of the consistent parameter set $\mathcal{P}_{c}$ such that for all $p \in \mathcal{P}_{i}$, the model is consistent with the measurements, i. e. $m_{y} \in \mathcal{M}$.

Fig. 1a describes the relation between the consistent parameter sets and outer and inner approximations thereof.

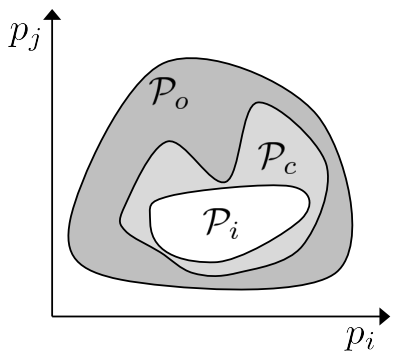

(a)

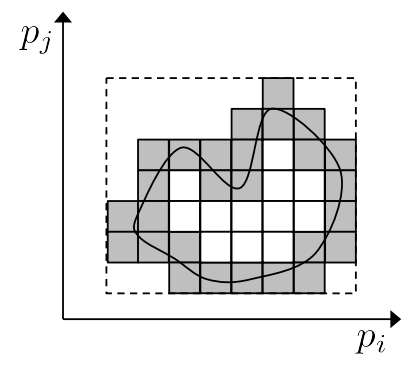

(b)
Fig. 1. (a) Illustration of relationships between outer $\mathcal{P}_{o}$ and inner approximation $\mathcal{P}_{i}$ of the consistent parameter set $\mathcal{P}_{c}$. (b) Algorithmic determination of outer and inner approximations. The dashed box shows a hyperrectangular outer approximation. The dark boxes show an outer approximation determined by testing the different regions whether they contain consistent parameters. The white boxes show an inner approximation guaranteed to contain only consistent parameters.

The proposed and novel approach to find inner approximations $\mathcal{P}_{i}$ basically consists of two steps. First, the constraints $g_{y}(\cdot)$ are reformulated using binary variables and by imposing suitable constraints on the binary variables. By this, one obtains a feasibility problem that involves binary variables and which solution set (in parameter space) equals the solution set of FP. The second and crucial step is the inversion of the logical constraints on the binary variables. Using a mixed-integer linear program (MILP) it can be checked whether the logical constraints are violated or not, which then allows one to determine inner approximations. The following sections present these steps in detail.

\section{CERTIFICATES FOR GUARANTEED SOLUTION SETS}

To estimate inner approximations of consistent parameter sets, this paper extends the work by Rumschinski et al. [2012], in which quantitative constraints and qualitative knowledge are formulated via binary variables and logical conditions. By inversion of the logical constraints and by proving infeasibility of the inverted feasibility problem, it can be guaranteed that no constraint is violated - which provides an inner approximation.

\subsection{Mixed-integer Reformulation of Constraints}

The key idea is to express constraints by means of logical relationships between additionally introduced binary variables $\phi_{i} \in\{0,1\}, i=1,2, \ldots, c_{m}$. In particular, we reformulate the quantitative measurement constraints $\mathcal{M}$ using this framework. The variable $\phi_{i}$ indicates whether the constraint $g_{y, i}$ is satisfied $\left(\phi_{i}=1\right)$ or not $\left(\phi_{i}=0\right)$. To this end, we introduce additional constraints such that $\phi_{i}$ takes the desired value. This can be achieved by (see Rumschinski et al. [2012] and references therein)

$$
\begin{aligned}
& \phi_{i} \geq \frac{g_{y, i}\left(m_{y}\right)}{M_{i}}, \\
& \phi_{i} \leq \frac{g_{y, i}\left(m_{y}\right)}{M_{i}}+1, \quad i=1,2, \ldots, c_{m},
\end{aligned}
$$

where $M_{i}>\max _{\forall m_{y} \in \mathcal{Y}}\left|g_{y, i}\left(m_{y}\right)\right|$. Note that $\phi_{i}$ can be both 0 and 1 if a solution satisfies $g_{y, i}(\cdot)=0$. However, this does not restrict the following analysis.

To consider more complicated sets $\mathcal{M}$ described by $c_{m}$ inequalities, it has to be demanded that all binary variables $\phi_{i}$ are equal to 1 . One thus obtains a linear constraint:

$$
\sum_{i=1}^{c_{m}} \phi_{i}=c_{m} .
$$

As can be easily seen, if any $\phi_{i}$ is 0 , then constraint (6) is not satisfied. The derived constraints therefore correspond to a conjunction, i. e. a logical and-combination of the binary variables.

The constraints (5) and (6) can be added to FP, which then is denoted by MIFP due to the integrality constraints involving the binary variables:

$$
\text { MIFP }: \begin{cases}\text { find } & \xi_{\text {MIFP }} \\ \text { s.t. } & \text { equations }(1), \\ & x(k) \in \mathcal{X}, \forall k \in \mathcal{T}, \\ & p \in \mathcal{P}, \\ & \text { equations }(5), \\ & \sum_{i=1}^{c_{m}} \phi_{i}=c_{m} .\end{cases}
$$

Then, one can state:

Lemma 1. (Equivalence of FP and MIFP). The solution sets in parameter space of $\mathrm{FP}$ and MIFP are equal, i. e. $\mathcal{P}_{c}^{\mathrm{FP}}=\mathcal{P}_{c}^{\mathrm{MIFP}}$.

Proof: The proof is obvious and follows from the conversion of the FP into a MIFP.

In Rumschinski et al. [2012], a theorem was presented giving inconsistency certificates based on the FP. Using Lemma 1 and the theorem from Rumschinski et al. [2012], one can now state (without the need of a formal proof):

Theorem 1. (MIFP inconsistency certificate). If MIFP does not admit a solution, then there exists no parametrization $p \in \mathcal{P}$ and corresponding state trajectory $x(k) \in \mathcal{X}$ such that $m_{y} \in \mathcal{M}$.

As mentioned in Section 2, it is difficult to check consistency or inconsistency of the FP or MIFP directly. However, by applying relaxations (cf. Section 2.2) to obtain a mixed-integer linear feasibility problem (MILP) and by using weak-duality and Theorem 1 , one is then able to derive outer approximations of consistent parameter sets.

Note that the reformulation into a MIFP results in an increased problem size in terms of the number of variables and constraints. To be more specific, there are $c_{m}$ additional binary variables $\phi_{i}, 2 c_{m}$ additional nonlinear constraints (5), and one constraint representing the combination of the binary variables (6). However, efficient and parallel implementations of branch-and-bound algorithms 
in state-of-the-art mixed-integer solvers are able to deal with it efficiently.

\subsection{Inner Approximations by Constraint-inversion}

Due to the convex relaxations into mixed-integer linear programs (MILP), it is likely to happen that the solution set increases such that it contains inconsistent parameter sets. Thus, it might happen that there are parameter values for which

$$
\sum_{i=1}^{c_{m}} \phi_{i} \leq c_{m}-1
$$

Note that constraint (8) corresponds to the inversion of the consistency constraint (6). The crucial idea is now to reformulate the MIFP and check whether parameters satisfying (8) exist. This is achieved by adding the inverted consistency constraint (8) to the FP instead of the original constraint (6) leading to

$$
\widehat{\operatorname{MIFP}}: \begin{cases}\text { find } & \xi_{\text {MIFP }} \\ \text { s.t. } & \text { equations }(1), \\ & x(k) \in \mathcal{X}, \forall k \in \mathcal{T}, \\ & p \in \mathcal{P}, \\ & \text { equations }(5), \\ & \sum_{i=1}^{c_{m}} \phi_{i} \leq c_{m}-1 .\end{cases}
$$

If one can show that $\widehat{\mathrm{MIFP}}$ is infeasible, then all parameter values $p \in \mathcal{P}$ are guaranteed consistent with the measurements $\mathcal{M}$. Before one can formulate this in a theorem, two assumptions are needed:

Assumption 1. (Existence of solutions). For every $p \in$ $\mathcal{P}$ there exists a solution $x(0), x(1), \ldots, x\left(n_{t}-1\right)$ and $y(0), y(1), \ldots, y\left(n_{t}-1\right)$.

This is a mild assumption which many systems fulfill, such as in particular polynomial systems given in the explicit form $x(k+1)=\tilde{F}(x(k), p)$.

Furthermore it has to be assumed:

Assumption 2. (Bounds on solutions). For all $p \in \mathcal{P}$, it holds that $x(k) \in \mathcal{X} \forall k \in \mathcal{T}$.

This assumption demands known guaranteed enclosures on the states on the time interval $\mathcal{T}$. Note that this is also a mild assumption because conservative bounds can be chosen for $\mathcal{X}$ or can be derived from system insight like mass conservation in biochemical reaction networks. The main purpose of Assumption 2 is to reduce the number of constraints that have to be tested for consistency. Then, one only needs to check and invert constraints $g_{y, i}$ and does not have to consider $g_{x, i}$ Eq. (4).

With $\widehat{\text { MILP }}$ denoting the mixed-integer linear relaxation of the mixed-integer nonlinear feasibility problem $\widehat{\mathrm{MIFP}}$, one is now able to state the following:

Theorem 2. (Consistency certificate). The model (1) is guaranteed consistent with the measurements $\left(m_{y} \in \mathcal{M}\right)$ for all $p \in \mathcal{P}$, if the Lagrangian-dual of the $\widehat{\text { MILP }}$ is unbounded.
Proof: The weak-duality theorem and the relaxation process guarantee that if the dual $\widehat{\mathrm{MILP}}$ is unbounded, then the $\widehat{\mathrm{MIFP}}$ does not admit a solution. Due to Assumptions 1 and 2 it follows that (8) is not satisfied, hence (6) is satisfied for all $p \in \mathcal{P}$.

By inversion of the logical representation of the measurement constraints (5) and (6), we were able to derive a theorem that can be used to address Problem 1.

\section{ALGORITHMS FOR INNER APPROXIMATIONS}

This section presents two algorithms to determine inner approximations $\mathcal{P}_{i}$ based on Theorem 2 . Since the theorem builds on infeasibility, i.e. no solution exists, it is not straightforward to use optimization that returns optimal feasible solutions. Instead, entire parameter regions are either recursively (Algorithm 1) or incrementally (Algorithm 2) proved to be an inner approximation by virtue of Theorem 2. Both algorithms terminate in finite time. Application of the algorithms is shown in the next section.

\subsection{Recursive Inner Approximation}

The following algorithm assumes that the initial parameter set $\mathcal{P}$ is given by lower bounds $\underline{p}_{j}$ and upper bounds $\bar{p}_{j}$ in the following form:

$$
\mathcal{P}:=\left\{\underline{p}_{j} \leq p_{j} \leq \bar{p}_{j}, j=1,2, \ldots, n_{p}\right\} .
$$

Eq. (10) represent a hyperrectangular description of the parameter uncertainties. The recursive Algorithm 1 uses bisectioning and it tests whether a hyperrectangle is an inner approximation or not. In case it is not, then the hyperrectangle is split into two parts along a heuristically or randomly chosen direction and the two obtained hyperrectangles are tested. The recursion terminates either if a hyperrectangle is proved an inner approximation, or if a predefined recursion depth $N_{\max }$ is reached.

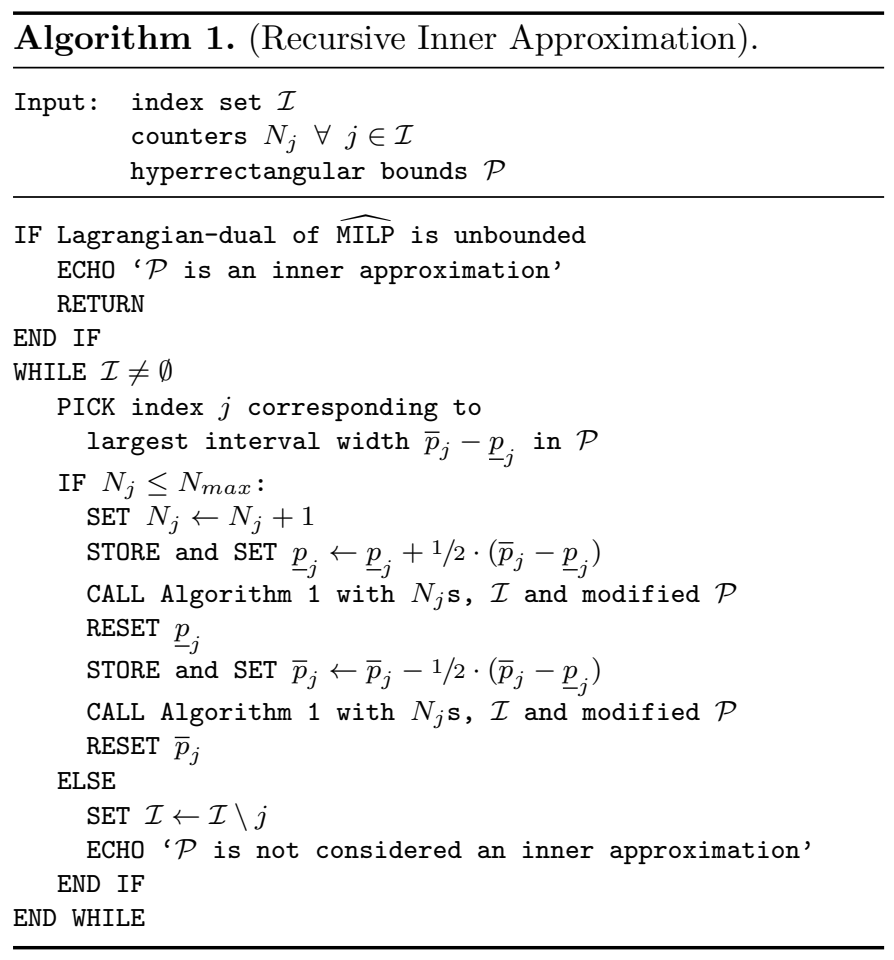

The algorithm is initially called with the parameter 
index set $\mathcal{I}:=\left\{1,2, \ldots, n_{p}\right\}$, a set of $n_{p}$ counters $N_{j}$ which are all set to 1 , and the initial uncertain parameter description (10).

The recursion depth $N_{\max }$ and the dimension of $\mathcal{P}$ determine the number of hyperrectangles to be checked and thereby the accuracy of the inner approximation. In general, the number of hyperrectangles grows exponentially, but the algorithm terminates after a finite number of steps. The algorithm is suited to explore the parameter space for inner approximations. However, it is computationally more demanding to prove a region to be an inner approximation than to check the same region whether it contains no solutions at all using certificates for outer approximations. It is therefore beneficial to combine both outer and inner approximations by, first, using certificates for outer approximations up to a certain recursion depth and, second, to check the remaining boxes whether they are valid inner approximations or not.

\subsection{Incremental Polytopic Expansion}

The motivation and starting point for the second algorithm is the fact that often consistent parameter values are available. Such parameter values could be obtained e.g. by Monte-Carlo sampling [Robert and Casella, 2005] or by local nonlinear optimization. The following algorithm relies on such samples and aims for a polytopic inner approximation. This is done by first testing whether the parameter set defined by the polytopic convex hull of the samples is an inner approximation or not. If this is the case, then the algorithm tries to get a bigger (in terms of its volume) inner approximation by expanding the polytope. This is done by incrementally moving the facets of the polytope outwards, thus increasing the volume of the polytope, until the inner approximation cannot be guaranteed.

Assume that a polytopic convex hull of a number of samples is given by

$$
\mathcal{P}:=\left\{p: a_{j}^{T} p \geq b_{j},\left\|a_{j}\right\|_{2}=1, \quad j=1,2, \ldots, c_{f}\right\},
$$

where $a_{j}$ are the (outwards-facing) unit normal vectors of the $c_{f}$ facets of the polytope. In addition, assume that $\mathcal{P}$ is bounded and represents a polytope of full dimension in $\mathbb{R}^{n_{p}}$. One can then propose:

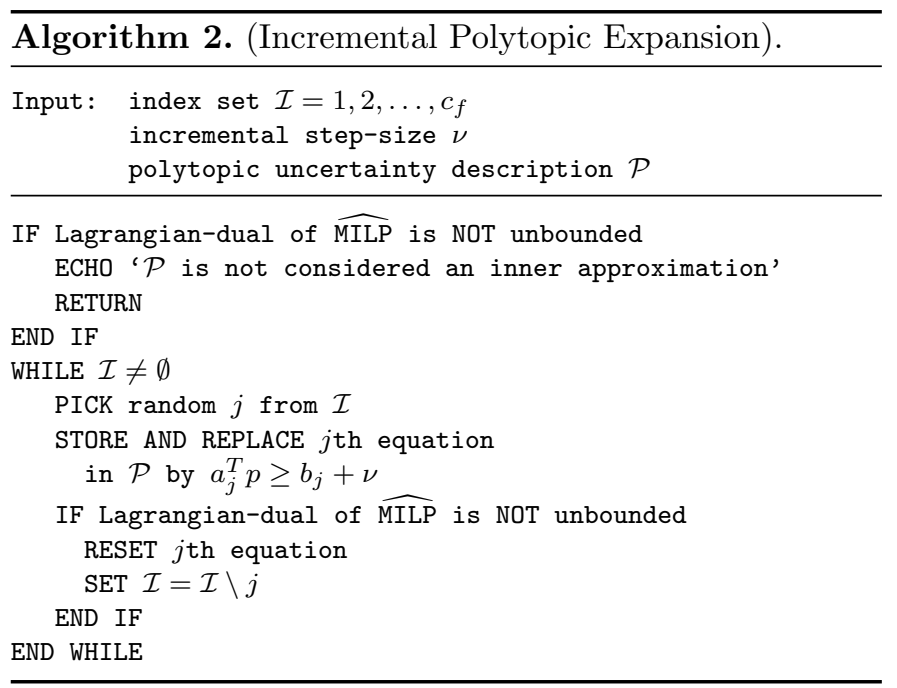

The algorithm terminates after a finite number of steps if the consistent parameter set is bounded. Due to the heuristics, such as the random choice of the next facets and the step size $\nu$, the obtained polytopic description of the inner approximation is not unique and might not be the optimum in terms of maximal volume.

Note that one might have to add additional checks in Algorithm 2 to verify that the shifting of a facet does not render this facet empty, i. e. leads to an inactive constraint.

If the polytope $\mathcal{P}$ has initially degenerated dimension, additional facets can be introduced heuristically. Note that also in the special case of only one consistent sample, the polytope to be expanded can e.g. be assumed an axesaligned hyperrectangle or hypercube with zero length. Then this hyperrectangle can be expanded similarly.

The advantage of Algorithm 2 is that once an inner approximation has been found based on samples, then no further sampling is required since it has been proved that the polytope describes an inner approximation.

\section{EXAMPLE}

For illustration, consider an enzyme-catalyzed reaction:

$$
\begin{aligned}
& S_{1}+E \underset{p_{2}}{\stackrel{p_{1}}{\rightleftharpoons}} C_{1} \stackrel{p_{3}}{\rightarrow} P+E \\
& S_{2}+E \underset{p_{5}}{\stackrel{p_{4}}{\rightleftharpoons}} C_{2} .
\end{aligned}
$$

Here, enzyme $E$ and a substrate $S_{1}$ reversibly form a complex $C_{1}$ that is converted into product $P$. Furthermore, the enzyme is bound by a second substrate $S_{2}$ forming the inhibitory complex $C_{2}$. The parameters $p_{1}, p_{2}, \ldots, p_{5}$ denote the unknown rate constants for which we aim to determine inner approximations. All results were obtained using the toolbox ADMIT [Streif et al., 2012].

\subsection{Model Description}

The reaction mechanism (12) is modeled by

$$
\begin{aligned}
& x_{1}(k+1)=x_{1}(k)+h\left(p_{1} x_{4}(k) x_{5}(k)-\left(p_{2}+p_{3}\right) x_{1}(k)\right) \\
& x_{2}(k+1)=x_{2}(k)+h\left(p_{4} x_{5}(k) x_{6}(k)-p_{5} x_{2}(k)\right) \\
& x_{3}(k+1)=x_{3}(k)+h\left(p_{3} x_{1}(k)\right),
\end{aligned}
$$

with $x_{1}(k), x_{2}(k), x_{3}(k), x_{4}(k), x_{5}(k)$ and $x_{6}(k)$ representing the concentrations of $C_{1}, C_{2}, P, S_{1}, E$ and $S_{2}$, respectively. The following conservation relationships hold: $x_{4}(k)+x_{1}(k)+x_{3}(k)=1, x_{5}(k)+x_{1}(k)+x_{2}(k)=1, x_{6}(k)+$ $x_{2}(k)=1$. Artificial measurements of the product $P$ were obtained from the simulation of the system with nominal initial condition $\left[x_{1}(0), x_{2}(0), x_{3}(0)\right]^{\mathrm{T}}=[0.10,0.10,0.05]^{\mathrm{T}}$ for a step size of $h=0.1$ hours, and all parameter values set to 2. To simulate data uncertainties, an absolute error of $5 \%$ was added. Initial parameter uncertainties were assumed as $0.1 \leq p_{j} \leq 10, j=1, \ldots, 5$.

\subsection{Results}

By applying Algorithm 1, we determined an inner approximation of the parameters by partitioning the parameter space into boxes and checking if $\widehat{\mathrm{MIFP}}$ admits a solution or not. Fig. 2a shows the obtained inner approximation which 


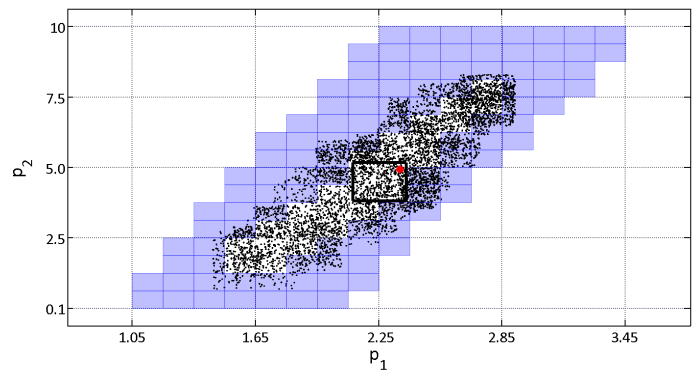

(a)

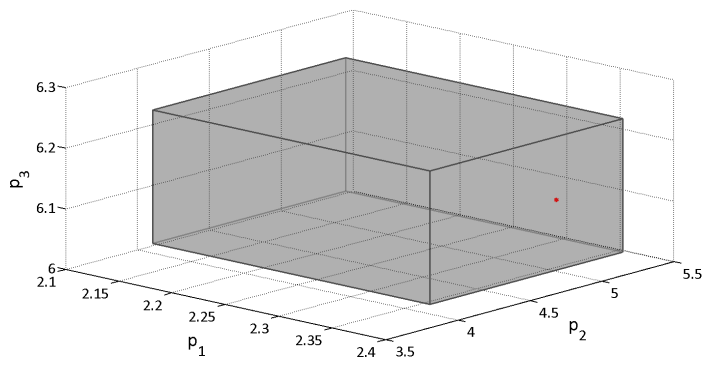

(b)

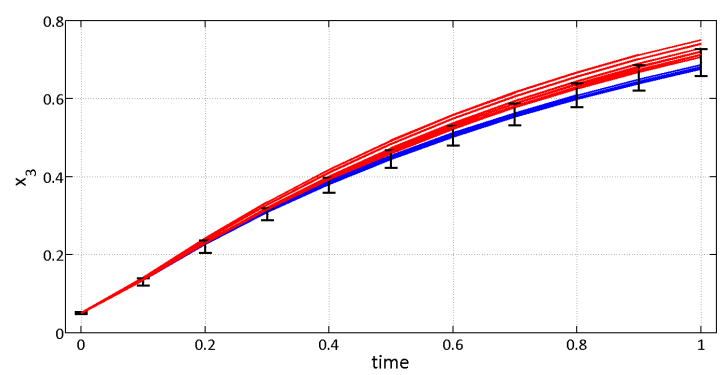

(c)

Fig. 2. (a) Estimation of $\mathcal{P}_{c}$ using bisectioning and Algorithm 1 An outer approximation $\mathcal{P}_{o}$ and inner approximation $\mathcal{P}_{i}$ are given by the light blue and white boxes, respectively. Black dots correspond to valid Monte-Carlo samples. The black rectangle shows the inner approximation obtained by Algorithm 2 (see also b) projected onto the $\left(p_{1}-p_{2}\right)$-space. (b) Polytopic/hyperrectangular expansion (using Algorithm 2) plotted in $\left(p_{1}-p_{2}-p_{3}\right)$-space starting with the valid sample $[2.36,4.98,6.12,1.62,2.98]$ (red dot) lead to the inner approximating hyperrectangle (gray box) $[2.13,2.39] \times[3.88,5.22] \times$ $[6.03,6.25] \times[1.45,1.76] \times[2.81,3.03]$. (c) Model simulations using parameter samples from the outer (red) and inner approximations (blue).

covers $82 \%$ of the valid Monte-Carlo samples. These results show that our approach yields good inner approximations.

Using Algorithm 2, we determined the polytope Fig. 2b starting with a single valid parameter sample (red dot) and setting $\nu=0.01$.

Fig. 2c shows the obtained time plots using parameter samples from the outer approximations (red) and the inner approximations (blue). The plots show that samples from the inner approximations lead to solutions such that initial constraints on e.g. artificially or experimentally determined measurements (given as error bars) are always satisfied. Samples from the outer approximations can lead to invalid solutions as constraints on data might be violated.

\section{CONCLUSIONS AND OUTLOOK}

This paper proposed a method for the estimation of inner approximations of polynomial discrete-time system. Inner approximations guarantee that for all possible parameter combinations within this set the considered constraints are satisfied. This enables one to make reliable modelbased predictions which play a significant role e. g. in the development of disease-specific therapies.

The presented approach leads to a problem that scales linearly with the number of constraints with respect to which an inner approximation is searched for. This might lead to an intractable problem if the number of constraints is large. As a remedy, the inner approximation can be refined by considering new or additional constraints or measurements in a recursive manner.

\section{REFERENCES}

Borchers, S., Rumschinski, P., Bosio, S., Weismantel, R., and Findeisen, R. (2009). A set-based framework for coherent model invalidation and parameter estimation of discrete time nonlinear systems. In Proc. Conference on Decision and Control (CDC), 6786-6792. Shanghai, China.

Jaulin, L. and Walter, E. (1993). Set inversion via interval analysis for nonlinear bounded-error estimation. Automatica, 29(4), 1053-1064.

Korda, M., Henrion, D., and Jones, C.N. (2012). Inner approximations of the region of attraction for polynomial dynamical systems. URL arXiv: 1210.3184.

Robert, C. and Casella, G. (2005). Monte Carlo Statistical Methods. Springer, New York, 2nd edition.

Rumschinski, P., Borchers, S., Bosio, S., Weismantel, R., and Findeisen, R. (2010). Set-based dynamical parameter estimation and model invalidation for biochemical reaction networks. BMC Syst. Biol., 4, 69-82.

Rumschinski, P., Streif, S., and Findeisen, R. (2012). Combining qualitative information and semi-quantitative data for guaranteed invalidation of biochemical network models. Int. J. Robust. Nonlinear Control, 22(10), 11571173 .

Streif, S., Savchenko, A., Rumschinski, P., Borchers, S., and Findeisen, R. (2012). ADMIT: a toolbox for guaranteed model invalidation, estimation and qualitativequantitative modeling. Bioinformatics, 28(9), 12901291.

Streif, S., Waldherr, S., Allgöwer, F., and Findeisen, R. (2009). Systems Analysis of Biological Networks, chapter Steady State Sensitivity Analysis of Biochemical Reaction Networks: a Brief Review and New Methods, 129-148. Methods in Bioengineering. Artech House MIT Press, Boston/London.

Streif, S., Rumschinski, P., Henrion, D., and Findeisen, R. (2013). Estimation of consistent parameter sets of nonlinear continuous-time systems using occupation measures and LMI relaxations. In Proc. Conference on Decision and Control $(C D C)$. Florence, Italy. To appear. Zhou, K., Doyle, J.C., and Glover, K. (1995). Robust and Optimal Control. Prentice Hall, Upper Saddle River, New Jersey. 